\title{
Selegiline remarkably improved stage 5 treatment- resistant major depressive disorder: a case report
}

\author{
This article was published in the following Dove Press journal: \\ Neuropsychiatric Disease and Treatment \\ 16 October 2013 \\ Number of times this article has been viewed
}

\author{
Yuji Kitaichi' \\ Takeshi Inoue' \\ Nobuyuki Mitsui' \\ Shin Nakagawa' \\ Rie Kameyama' \\ Yoshiyuki Hayashishita' \\ Tohru Shiga ${ }^{2}$ \\ Ichiro Kusumi' \\ Tsukasa Koyama' \\ 'Department of Psychiatry, \\ Graduate School of Medicine, \\ Hokkaido University, Sapporo, Japan; \\ ${ }^{2}$ Department of Nuclear Medicine, \\ Graduate School of Medicine, \\ Hokkaido University, Sapporo, Japan
}

Correspondence: Yuji Kitaichi Department of Psychiatry, Hokkaido University Graduate School of Medicine, North 15, West 7, Kita-ku,

Sapporo 060-8638, Japan

$\mathrm{Tel}+8 \mathrm{I}$ II 7065160

Fax +8I I| 706508 |

Email muenchen-yuji@huhp.hokudai.ac.jp

\begin{abstract}
We report a case in which selegiline, an irreversible monoamine oxidase B (MAO-B) inhibitor, greatly improved depressive symptoms in an adult with stage 5 treatment-resistant major depressive disorder. Four antidepressants and four augmentation therapies had previously been ineffective or intolerable, and electroconvulsive therapy had only a temporary effect. After 20 weeks of treatment with selegiline ( $10 \mathrm{mg}$ /day), the patient's score on the 17-item Hamilton Depression Rating Scale (HDRS) had decreased from 19 to 4 points. $\left[{ }^{18} \mathrm{~F}\right]$-Fluorodeoxyglucose positron emission tomography (FDG-PET) showed increased glucose metabolism in the bilateral basal ganglia after initiating selegiline treatment; blood dopamine levels were also increased after selegiline treatment. These results raise the possibility that selegiline enhances dopaminergic neural transmission in treatment-resistant depression, thus leading to an improvement in depressive symptoms.
\end{abstract}

Keywords: treatment-resistant depression, FDG-PET, glucose metabolism, basal ganglia

\section{Introduction}

Transdermal selegiline, an irreversible selective monoamine oxidase B (MAO-B) inhibitor, is approved as an antidepressant in the USA. ${ }^{1}$ Some reports have shown that oral selegiline treatment can also be effective in treating depression. For example, oral selegiline was effective for treatment-resistant elderly depressive patients at high doses $^{2}$ and exhibited antidepressant effects on severe refractory depression at more typical dosing regimens. ${ }^{3}$ On the other hand, one report indicates that low-dose transdermal selegiline treatment, but not oral selegiline treatment, was effective for treatment-resistant depression. ${ }^{4}$

We experienced a patient with severe stage 5 treatment-resistant major depression, which is defined by the persistence of significant or moderate depressive symptoms despite at least two treatment trials with antidepressants from different pharmacological classes and two augmentation therapies, as well as electroconvulsive therapy (ECT), as classified by Thase and Rush. ${ }^{5}$ Because selegiline may enhance dopaminergic neural transmission, ${ }^{6}\left[{ }^{18} \mathrm{~F}\right]$-fluorodeoxyglucose positron emission tomography (FDG-PET) of his brain was performed, and blood levels of dopamine, noradrenalin, and homovanillic acid (HVA) were measured. In addition, ß-phenylethylamine (PEA) levels in the patient's urine were measured because selegiline increases PEA levels through MAO-B inhibition. PEA stimulates trace amine-associated receptors (TAAR), which respond to trace amines, p-tyramine, and PEA, but not classic biogenic amines. ${ }^{7}$ 


\section{Case description}

A 51-year-old man had been diagnosed with major depression 6 years prior to beginning selegiline treatment. During that time, he was treated with imipramine, a tricyclic antidepressant (TCA) (150 mg/day), and his symptoms remitted. However, although he continued medication for 4 years, his depression relapsed. Imipramine was thus replaced with milnacipran, a serotonin and noradrenalin reuptake inhibitor $(150 \mathrm{mg} /$ day $)$ and then paroxetine, a selective serotonin reuptake inhibitor ( $40 \mathrm{mg} /$ day), but those were ineffective, and he was later admitted to our hospital. Concurrent administration of amoxapine, a tetracyclic antidepressant (TeCA) (150 mg/day), lithium carbonate ( $800 \mathrm{mg} /$ day), and cabergoline $(1 \mathrm{mg} / \mathrm{kg})$ improved his symptoms, and he was discharged. His plasma lithium concentration was $0.61 \mathrm{mEq} / \mathrm{L}$. However, he again relapsed into depression 8 months later, despite receiving treatment. His major symptoms included fatigue, loss of motivation, and decline in work efficacy. Subsequently, he was readmitted to our hospital.

During this second hospitalization, amoxapine (200 mg/day), amitriptyline, a TCA (200 mg/day), imipramine $(250 \mathrm{mg} /$ day $)$, and clomipramine, a TCA $(150 \mathrm{mg} /$ day) were consecutively used for at least 4 weeks, but none were effective. Additional lithium augmentation had no effect, and the addition of olanzapine $(5 \mathrm{mg} /$ day) was ineffective. Likewise, pramipexole $(2.625 \mathrm{mg} /$ day $)$ induced the side effects of oral dyskinesia and auditory hyperesthesia, while the addition of triiodothyronine was stopped because of palpitations. ECT was administered ten times, and depressive symptoms disappeared transiently. However, the patient's depression relapsed again 1.5 months later, despite the continuation of clomipramine after ECT. Selegiline $(2.5 \mathrm{mg} /$ day $)$ treatment was started after he signed the informed consent form. The daily dose of selegiline was increased by $2.5 \mathrm{mg}$ every week up to a maximum of $10 \mathrm{mg} /$ day. Mianserin, a TeCA, (60 mg/day), and valproate $(600 \mathrm{mg} /$ day) were also concurrently used, but the dosages of those drugs remained constant during selegiline treatment. Before initiating selegiline treatment, the patient received a score of 19 on the 17-item Hamilton Depression Rating Scale (HDRS). During selegiline treatment, his score on the HDRS decreased to 9 points at week 8 and 4 points at week 20 . He returned to work after 49 weeks of selegiline treatment. No side effects from selegiline treatment were either observed or reported. We followed up with the patient approximately 2 years and 5 months after the trial with selegiline. During this period, no relapse occurred.
FDG-PET scans were measured twice, before selegiline treatment and after 20 weeks of selegiline treatment. Two experienced nuclear medicine physicians visually interpreted the images. Twenty weeks after selegiline treatment, selegiline-induced enhancement of glucose metabolism was observed in the bilateral basal ganglia by FDG-PET, while decreases were shown in those in the occipital lobe (Figure 1). The methods used to conduct and analyze the PET scan were as follows: a 10-minute regional static scan was performed with a Siemens ECAT HR+ scanner using the three-dimensional (3D) mode, and the image was reconstructed in the brain mode, using Siemens' ecat software, version 7.2.2 (Siemens AG, Munich, Germany). The images were acquired via 3 minutes of transmission scanning and 10 minutes of emission scanning. The energy window was $350-650 \mathrm{keV}$. In the brain mode, the acquired 3D sonograms were converted into two-dimensional (2D) sonograms, using Fourier rebinning. The images were reconstructed using the direct inversion Fourier transformation method. We used a Hanning reconstruction filter with a $4 \mathrm{~mm}$ full width at half-maximum (FWHM). The reconstruction matrix was $256 \times 256$, and the field of view was $33 \mathrm{~cm}$ in diameter. The FWHM was $6.4 \mathrm{~mm}$ after reconstruction.

Moreover, the patient's plasma dopamine levels ( $<5$ to $9 \mathrm{pg} / \mathrm{mL}$ ) and urine PEA levels ( $<0.5$ to $179.4 \mathrm{ng} / \mathrm{mg}$ CRE [creatinine]) were greater after 12 weeks of selegiline treatment compared with baseline, although the plasma HVA and noradrenalin levels were not changed. The MAO-B activity

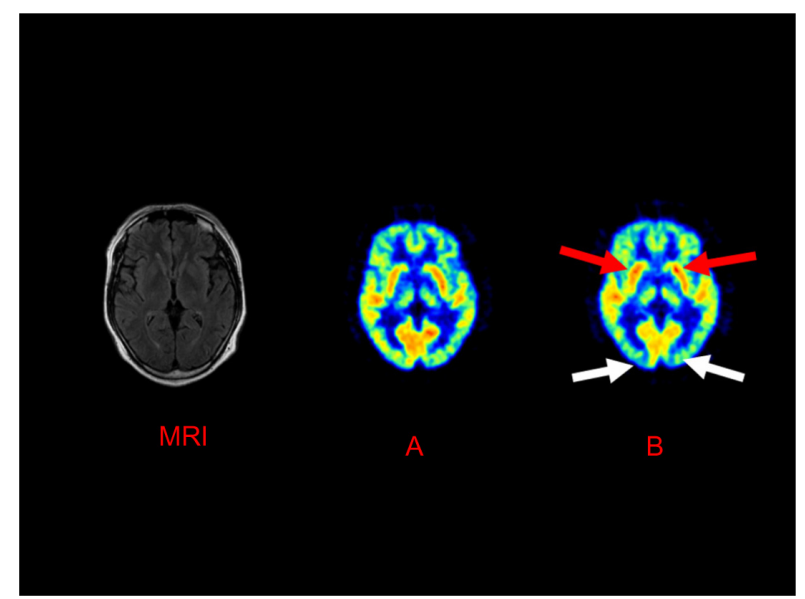

Figure I The changes in glucose metabolism using FDG-PET before and after selegiline treatment.

Notes: (A) Before selegiline treatment, the patient's 17-item HDRS score was 19 points; (B) after 20 weeks of selegiline treatment, a 17-item HDRS score was 4 points. Selegiline treatment increased glucose metabolism in the bilateral basal ganglia (red arrows) and decreased glucose metabolism in the occipital lobe (white arrows).

Abbreviations: FDG-PET, $\left[{ }^{18} \mathrm{~F}\right]$-fluorodeoxyglucose positron emission tomography; HDRS, Hamilton Depression Rating Scale; MRI, magnetic resonance imaging. 
of blood platelets was nearly completely inhibited by administering selegiline $10 \mathrm{mg} /$ day.

\section{Discussion}

In the history of this case, various antidepressants were tried, ultimately without success. Lithium and olanzapine augmentation therapies, for example, were ineffective. The addition of a dopamine agonist or triiodothyronine had been intolerable, and ECT showed only transient effects. In short, this case was considered to be a stage 5 treatmentresistant major depressive disorder, as classified by Thase and Rush. ${ }^{5}$ There have been few studies of stage 5 . This is the first report of successful selegiline treatment for a stage 5 treatment-resistant major depressive patient and of the FDGPET time course before and after selegiline.

Increases in the glucose metabolism of the bilateral basal ganglia and decreases in those of the occipital lobe were observed using FDG-PET, after selegiline treatment.

Recently, two FDG-PET studies ${ }^{8,9}$ reported different results from ours, showing that repetitive transcranial magnetic stimulation decreased abnormally elevated glucose metabolism in the left temporal cortex and fusiform gyrus, in persons with stage 2 major depressive disorder, which is defined as insufficient improvement despite at least two antidepressant trials ${ }^{8}$ and the presence of increased glucose metabolism in subdivisions of the anterior cingulate cortex (Brodmann areas 24 and 32). ${ }^{9}$ Differences in the staging of treatment resistance and in the studied treatments between our study and previous studies may account for the inconsistencies in the FDG-PET data. Nevertheless, the changes in glucose metabolism after selegiline treatment found in our study suggest that such changes are involved in the treatment response for persons with stage 5 major depression.

The main pharmacological functions of selegiline are (1) the reinforcement of dopamine transmission by MAO-B inhibition and (2) neuroprotective action. ${ }^{10}$ Because dopamine is abundant in the basal ganglia, the increases in glucose metabolism in the basal ganglia may occur via increases in dopamine by MAO-B inhibition after selegiline treatment. Moreover, a previous study has reported that volumetric decreases and regional blood flow decreases in the basal ganglia occur in major depressive disorders. ${ }^{11}$ However, there have been no studies about the effect of selegiline on FDGPET in Parkinson's disease or other diseases. An earlier study has suggested that the antidepressant effects in poststroke depression, in which biogenic amine-containing axons may be interrupted by ischemic lesions, may extend beyond mood symptoms, eg, to motor recovery. ${ }^{12}$ In addition, depressive symptoms are common in patients with Parkinson's disease, and improvement in mood, motor symptoms, and cognitive symptoms are found after the treatment of depression. ${ }^{13}$ These findings suggest that selegiline may not only improve mood, but also affect motor function. On the other hand, research using an animal model reported that selectively bred helpless rats treated with selegiline were no longer helpless when the tests were repeated. ${ }^{14}$ This suggests that selegiline also participates in the learning process. ${ }^{14}$ Although the glucose metabolism of the occipital lobe was decreased after selegiline treatment in this case, other antidepressant treatments, cognitive behavior therapy, and venlafaxine reportedly have increased the glucose metabolism of the occipital lobe. ${ }^{15}$ Thus, there seems to be a difference between selegiline and the conventional treatment for major depression. It is uncertain whether the decreased glucose metabolism in the occipital lobe in this case is related to the clinical efficacy of selegiline.

In addition to the FDG-PET data, increased plasma dopamine levels were also observed after selegiline treatment. Previously, we reported that a dopamine agonist (pramipexole) was effective for stage 2 treatment-resistant major depression; ${ }^{16}$ those results suggested that dopamine neurotransmission might be involved in treatment-resistant depression. Although it is not clear whether blood dopamine levels reflect dopaminergic neural transmission in the central nervous system, this dopaminergic effect may reflect MAO-B inhibition in the whole body, including the brain. Moreover, PEA levels in the urine of our patient were increased due to MAO-B inhibition by selegiline. PEA increases extracellular dopamine levels in the brain ${ }^{6}$ and acts at TAAR as an agonist. ${ }^{7}$ Together, these results raise the possibility that selegiline enhances dopaminergic neural transmission through one or more mechanisms, contributing to an overall antidepressive effect.

One limitation of this case report must be noted. Because selegiline had been added to mianserin and valproate, we cannot eliminate the possibility that the combined treatment was completely effective.

In conclusion, we reported on the progress of a stage 5 treatment-resistant major depressive patient, for whom selegiline greatly improved depressive symptoms. These results raise the possibility that selegiline produces antidepressant effects by enhancing dopaminergic neural transmission in the basal ganglia.

\section{Disclosure}

The authors report no conflicts of interest in this work. 


\section{References}

1. Bodkin JA, Amsterdam JD. Transdermal selegiline in major depression: a double-blind, placebo-controlled, parallel-group study in outpatients. Am J Psychiatry. 2002;159(11):1869-1875.

2. Sunderland T, Cohen RM, Molchan S, et al. High-dose selegiline in treatment-resistant older depressive patients. Arch Gen Psychiatry. 1994;51(8):607-615.

3. Higuchi H, Kamata M, Sugawara Y, et al. Remarkable effect of selegiline (L-deprenyl), a selective monoamine oxidase type-B inhibitor, in a patient with severe refractory depression: a case report. Clin Neuropharmacol. 2005;28(4):191-192.

4. Morgan PT. Treatment-resistant depression: response to low-dose transdermal but not oral selegiline. J Clin Psychopharmacol. 2007;27(3): 313-314.

5. Thase ME, Rush AJ. Treatment-resistant depression. In: Bloom FE, Kupfer DJ, editors. Psychopharmacology: The Fourth Generation of Progress. New York, NY: Raven Press; 2005:1081-1097.

6. Magyar K, Pálfi M, Tábi T, Kalász H, Szende B, Szöko E. Pharmacological aspects of (-)-deprenyl. Curr Med Chem. 2004;11(15):2017-2031.

7. Zucchi R, Chiellini G, Scanlan TS, Grandy DK. Trace amine-associated receptors and their ligands. Br J Pharmacol. 2006;149(8):967-978.

8. Li CT, Wang SJ, Hirvonen J, et al. Antidepressant mechanism of add-on repetitive transcranial magnetic stimulation in medicationresistant depression using cerebral glucose metabolism. J Affect Disord. 2010;127(1-3):219-229.
9. Baeken C, De Raedt R, Van Hove C, et al. HF-rTMS treatment in medication-resistant melancholic depression: results from 18FDG-PET brain imaging. CNS Spectr. 2009;14(8):439-448.

10. Youdim MB, Bakhle YS. Monoamine oxidase: isoforms and inhibitors in Parkinson's disease and depressive illness. Br J Pharmacol. 2006; 147 Suppl 1:S287-S296.

11. Canbeyli R. Sensorimotor modulation of mood and depression: an integrative review. Behav Brain Res. 2010;207(2):249-264.

12. Loubinoux I, Kronenberg G, Endres M, et al. Post-stroke depression: mechanisms, translation and therapy. J Cell Mol Med. 2012;16(9): 1961-1969.

13. Leentjens AF. The role of dopamine agonists in the treatment of depression in patients with Parkinson's disease: a systematic review. Drugs. 2011;71(3):273-286.

14. Schulz D, Mirrione MM, Henn FA. Cognitive aspects of congenital learned helplessness and its reversal by the monoamine oxidase (MAO)-B inhibitor deprenyl. Neurobiol Learn Mem. 2010;93(2): 291-301.

15. Kennedy SH, Konarski JZ, Segal ZV, et al. Differences in brain glucose metabolism between responders to CBT and venlafaxine in a 16-week randomized controlled trial. Am J Psychiatry. 2007;164(5): $778-788$.

16. Inoue T, Kitaichi Y, Masui T, et al. Pramipexole for stage 2 treatmentresistant major depression: an open study. Prog Neuropsychopharmacol Biol Psychiatry. 2010;34(8):1446-1449.
Neuropsychiatric Disease and Treatment

\section{Publish your work in this journal}

Neuropsychiatric Disease and Treatment is an international, peerreviewed journal of clinical therapeutics and pharmacology focusing on concise rapid reporting of clinical or pre-clinical studies on a range of neuropsychiatric and neurological disorders. This journal is indexed on PubMed Central, the 'PsycINFO' database and CAS.

\section{Dovepress}

The manuscript management system is completely online and includes a very quick and fair peer-review system, which is all easy to use. Visit http://www.dovepress.com/testimonials.php to read real quotes from published authors. 